\section{Vol No: 4, Issue: 2}

Received Date: Jun 10, 2019

Published Date: Jul 1, 2019

\section{Salvador Alvarez Ibarra}

Paloma Galan Solano

\section{Guillermo Padron Arredondo}

Department of General Surgery, General de Zona Hospital, Mexico

\section{Corresponding Author:}

\section{Salvador Alvarez Ibarra*}

Department of General Surgery, General de Zona Hospital \# 18 IMSS, Av. Tecnológico con Av. Petempich s/n Fraccionamiento Villamar II. Playa del Carmen, Quintana Roo. CP. 77711, Mexico.

\title{
Acute Intestinal Ischemia Due to Thrombosis of the Superior Mesenteric Artery in a Female Young Patient: A Clinical Case
}

\author{
ABSTRACT: \\ Introduction: Acute mesenteric ischemia is deadly from $50 \%$ to $90 \%$ of cases due to \\ poor understanding of the clinical picture of abdominal pain and the differential \\ diagnosis when it is not suspected and partly because of an unacceptable delay in \\ making the diagnosis.
}

Clinical case: A 31-year-old female with generalized abdominal pain without peritoneal irritation accompanied by vomiting and loose bowel movements. Vital signs: BP 90/70 mmHg, HR 100/min, BF 20/min, Temperature $96.8^{\circ} \mathrm{F}$. Laboratory test: Bh: Hto. 39.4, 42.6/103 leukocytes/uL, 89\% neutrophils, 10\% lymphocytes; QS: glucose $226 \mathrm{mg} / \mathrm{dL}$. It is surgically intervenes finding intestinal ischemia to $10 \mathrm{~cm}$ from the ligament of Treitz to $1 \mathrm{~m}$ from the ileocecal valve, the root of the mesentery is explored and superior mesenteric artery is explored obtain feeling weak pulse. Thrombectomy is performed with infusion of $1000 \mathrm{IU}$ of heparin in saline solution return to viability of ischemic bowel loops in $50 \%$ viable resulting $50 \mathrm{~cm}$ from the ligament of Treitz to $2 \mathrm{~m}$ from the ileocecal valve; in nonviable bowel segment resection is performed.

Discussion: The clinical diagnosis of mesenteric ischemia is difficult, and in most cases the abdominal pain is the cardinal symptom. The basis of the proper treatment of this entity is: early diagnosis, resection of the damaged intestine, restoration surgical blood flows or nonsurgical, second-look laparotomy and support of the ICU, as handled case described here.

Keywords: Acute Mesenteric Ischemia; Superior Mesenteric Artery Thrombosis; Diagnosis; Treatment; Prognosis; Biological Markers.

\section{INTRODUCTION}

Mesenteric ischemia is classified as acute and chronic and the first situation is an emergency that endangers life because the sudden decrease in blood flow can ultimately result in an intestinal infarction. The most common causes are: arterial embolism, arterial thrombosis, non-occlusive mesenteric ischemia and mesenteric venous thrombosis.

Acute mesenteric ischemia is fatal between $50 \%$ and $70 \%$ of cases due to poor understanding of the clinical picture of abdominal pain and its differential diagnosis when this is not suspected and, on the other hand, due to an unacceptable delay in making the precise diagnosis. The average diagnosis time is 7.9 hours and the 
treatment time is 2.5 hours before reperfusion is acquired. However, with a 6-hour ischemia, the disintegration of the mucosal barrier begins, producing translocation followed by morphological transformation of the intestinal wall.

While only $1 \%$ of cases of acute abdomen correspond to intestinal ischemia, this corresponds to $10 \%$ of cases of acute abdomen in those over 70 years. The main risk factors are: heart failure, atrial fibrillation, coronary artery disease, arterial hypertension and peripheral arterial occlusion. The risk factors that affect morbidity and mortality in acute mesenteric ischemia are: advanced age, leukocytosis, the time elapsed for laparotomy and colonic involvement.

Patients with a history of arterial embolism, vasculitis, deep vein thrombosis, hypercoagulable states or chronic postprandial pain are at greater risk of ischemia. In addition, vasculitis is a common cause of mesenteric ischemia in young patients with autoimmune diseases [1-4].

\section{CLINICAL CASE}

This is a 31-year-old female patient who goes to the emergency department for generalized abdominal pain without evidence of peritoneal irritation accompanied by vomiting of gastric contents and diarrhea without mucus or blood, without fever. He has a history of intestinal resection due to small bowel thrombosis a year ago with positive smoking 1/2 pack a day. Not diabetic or hypertensive. Vital signs of admission:BP 90/70 $\mathrm{mmHg}$, HR 100/min, HR 20/min, Temp. $36^{\circ} \mathrm{C}$. Laboratories: Bh: Hto. 39.4, leukocytes $42.6103 / \mu \mathrm{L}$, Neutrophils 89\%, lymphocytes 10\%; blood chemistry: Glucose $226 \mathrm{mg} / \mathrm{dL}$, hepatic function test, partial time of protrombine and partiel time of tromboplastine were normals. Imaging: simple radiographs of the standing and decubitus abdomen showing sentinel loop with air-fluid level, with normal ultrrasound and diagnostic CT Scanner for appendicitis. A physical examination of the abdomen painful to superficial, middle and deep palpation with data of peritoneal irritation, diminished peristalsis, signs of McBurney and psoas and positive talopercussion integrating the diagnosis of acute appendicitis modified by medications. Surgically intervened by finding intestinal ischemia $10 \mathrm{~cm}$ from the ligament of Treitz up to $1 \mathrm{~m}$ from the ileocecal valve (Figure 1), the root of the mesentery is explored by dissecting the Superior Mesenteric Artery (AMS) by palpating its weak pulse. Thrombectomy is performed with infusion of 1000 IU of heparin in saline with recovery of the viability of ischemic intestinal loops in $50 \%$ resulting in $50 \mathrm{~cm}$ viable from the ligament of Treitz and up to $2 \mathrm{~m}$ from the ileocecal valve; intestinal resection of the non-viable segment was performed with a terminal-terminal anastomosis in two planes (Figure 2). An incidental appendectomy was performed and the Bogota bag was left and the Intensive Therapy Unit moved. Second-look laparotomy is performed without setbacks at 24 hours. Immunological tests are performed due to previous ischemic history, resulting only in antinuclear antibodies (ANA) (+) 1:1000 ( $\mathrm{N}=<1: 100)$. The inpatient stay was nine days with high without complications. The patient is not followed up because she returned to her place of origin.

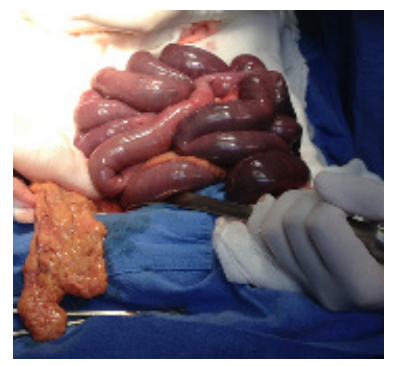

Figure 1: Healthy and ischemic small bowel.

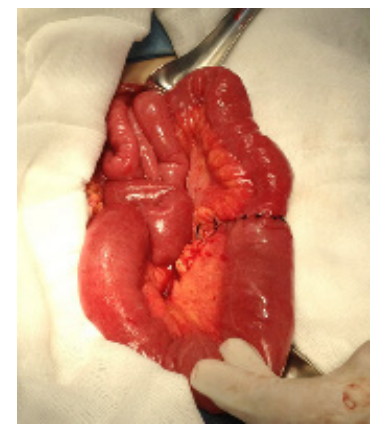

Figure 2: Term-terminal anastomosis.

\section{DISCUSSION}

The clinical diagnosis of mesenteric ischemia is difficult especially in the young patient, but in most cases abdominal pain is the cardinal symptom in $94 \%$, accompanied by nausea $56 \%$, vomiting $38 \%$, diarrhea $31 \%$, and tachycardia $31 \%$. It has been estimated that in most cases of intestinal ischemia (65\%) are caused by embolism or thrombosis and damage to the blood flow of the superior mesenteric artery affecting all or some proportion of the small intestine and the right colon. However imaging studies help to specify this diagnosis, it is known that radiological images are different in these cases when differentiating the etiology of the vascular event; For example, in the arterial etiology, as in this case, the progression of the 
damage is slower than in the ischemia of venous origin and the thinning of the intestinal wall is typical but difficult to recognize, hence its diagnosis is difficult. On the contrary, in ischemia of venous origin the progression of the damage is faster even when the symptomatology is less dramatic and the thickening of the intestinal wall is easy to observe and detect, simplifying its diagnosis.

Currently, the modality of reference for the diagnosis of intestinal ischemia is the contrasted tomography (sensitivity from $82 \%$ to $96 \%$ and specificity of $94 \%$ [gold standard]); however, there are some disadvantages with this diagnostic method such as: exposure to radiation, nephrotoxicity and possible allergic reaction to the contrast medium. Therefore, not all patients with suspected intestinal ischemia should undergo this study initially; however, despite its limitations, ultrasound could be a good method of study as the first choice in these patients.

Another current diagnostic modality is multidetection tomography angiography (MDCT) with which thickening of the intestinal wall, pneumatosis and intestinal dilation can be observed; as well as vascular occlusion, portomesenteric venous gas, mesenteric congestion and cavity free air. This study would be first choice in hospitals that have this equipment since it has a sensitivity of 64 to $93 \%$, specificity of $92 \%$ to $100 \%$, positive predictive value of $90 \%$ to $100 \%$ and negative predictive value of $94 \%$ to $98 \%[5-8]$.

A study by Wong Y et al., [10] was carried out with the purpose of detecting the venous or arterial origin of mesenteric vascular occlusion by means of secondary findings and the independent findings of the CT that suggested very serious events, finding that the secondary findings of the CT scan They are presented with different frequencies between thromboembolism of the superior mesenteric artery (TEAMS) and thrombosis of the superior mesenteric vein (SMVT), finding that the reduction of intestinal wall improvement, paralytic ileus, and intestinal pneumatosis are more frequent in the involvement of the TEAMS and, on the contrary, the thickening of the intestinal wall, the edema of the mesentery and the ascites are frequent in the TVMS. On the other hand, the independent findings that indicate very serious mesenteric occlusion are the decrease in the enhancement of the intestinal wall and the paralytic ileus.

Because not all hospitals are equipped with tomography, some researchers have tried other methods to integrate the diagnosis of mesenteric ischemia, $\mathrm{Oz} \mathrm{B}$, et al., [9] conducted a study on gastric tonometry without obtaining favorable results with this method.

Because diagnosis is vital to initiate treatment, new diagnostic strategies are required, so some simple noninvasive biochemical tests could increase the clinical suspicion of acute mesenteric ischemia (AMI) and help improve the selection of patients before submitting them. To radiographic studies, for which the IMA could be diagnosed early and followed up by CT angiography or MRI. Recent experimental studies are promising for alphaglutathione S transferase (GST) and fatty acids bound to intestinal proteins (I-FABP) as early markers of intestinal ischemia with good prospects [10-12].

Jun KW, et al. [13] in one of their studies report that the bases for the adequate treatment of this entity are: early diagnosis, resection of the damaged intestine, restoration of surgical or non-surgical blood flow, second-look laparotomy and support of the Therapy Unit Intensive, as was the case described here. In selected cases and with the necessary supplies, endovascular therapy with self-expandable stenting is a good treatment option $[14,15]$

In order to know the prognosis of these patients, Altintoprak F, et al., [16] studied the importance of mean platelet volume (PMV), which can be predictive for patients with poor prognosis and for the planning of surgical reoperations. This prognostic factor is higher in patients who die. Romano $\mathrm{N}$, et al., [17] reported a case of acute thrombosis of the superior mesenteric artery (TAAMS) in a young woman with factor $S$ deficiency, which is why they recommend that although mesenteric infarction has a low incidence in these patients, thrombosis acute should be suspected, especially in patients who are receiving estrogen and progestin and who present with acute abdominal pain.

Winkel N, et al., [18] report a case of segmental occlusion of the superior mesenteric artery (AMS) that caused mesenteric infarction diagnosed by CT, enteroscopy and digital subtraction angiography intra-arterial, which revealed the occlusion of a branch of the artery. AMS with collateral vascularity; an enteroclysis confirmed the diagnosis.

With respect to the presence of antinuclear antibodies in the blood, this may be due to: chronic liver disease, collagen vascular disease, medication-induced lupus erythematosus, myositis (inflammatory muscle disease), rheumatoid arthritis, Sjogren's syndrome, and systemic lupus erythematosus. The increase in antinuclear antibody levels can be observed, sometimes, 
in people with: systemic sclerosis (scleroderma), and thyroid disease. In this case, the antinuclear antibodies were high and the rest of the negative tests, however, the case was not followed up due to loss of the patient.

\section{CONCLUSIONS}

The mortality rate in intestinal ischemia is high; patients with risk profiles should suspect the diagnosis; As soon as there is suspicion, treatment should be immediate as if it were a vascular emergency similar to myocardial infarction; in cases of suspected thromboembolism, a biphasic CT is indicated, and in cases of peritonitis, portions of the intestine with irreversible necrosis should be resected plus surgical revascularization.

\section{REFERENCES}

1. Walker TG. (2009). Mesenteric ischemia. Sem Intervent Radiol. 26(3): 175-183.

2. Klar E, Rahmanian PB, Bucker A, Hauenstein $\mathrm{K}$, et al. (2012). Acute Mesenteric ischemia: a vascular emergency. Dtsch Arztebl Int. 109(14): 249-256.

3. Aliosmanoglu I, Gul M, Kapan M, Arikanoglu Z, et al. (2013). Risk factors effecting mortality in acute mesenteric ischemia and mortality rates: a single center experience. Int Surg. 98(1): 76-81.

4. Hokama A, Kishimoto K, Ihama Y, Kobashigawa Ch, et al. (2012). Endoscopic and radiographic features of gastrointestinal involvement in vasculitis. World $\mathrm{J}$ Gastroint Endosc. 4(3): 50-56.

5. Reginelli A, Iacobellis F, Berritto D, Fonio P, et al. (2013). Mesenteric ischemia: the importance of differential diagnosis for the surgeon. BMC Surg. 13(Suppl 2): S51.

6. Reginelli A, Genovese E, Cappabianca S, Iacobellis F, et al. (2013). Intestinal Ischemia: US-CT findings correlations. Crit Ultrasound J. 5(Suppl 1): S7.

7. Costa AF, Chidambaram V, Lee JJ, Asquith J, et al. (2014). Multidetector computed tomography of mesenteric ischaemia. Insights Imaging. 5(6): 657-666.

8. Khoshini R, Garrett B, Sial S and Eysselein VE. (2004). The role of radiologic studies in the diagnosis of mesenteric ischemia. MedGenMed. 6(1): 23.

9. Oz B, Akyuz M, Emek E, Soziuer E, et al. (2014). The effectiveness of gastric tonometry in the diagnosis of acute mesenteric ischemia in cases where a contrastenhanced computed tomography cannot be obtained. Ulus Cerrahi Derg. 31(1): 26-29.

10. Wong YC, Wu CH, Wang LJ, Chen HW, et al. (2013). Mesenteric vascular occlusion: comparison of ancillary CT findings between arterial and venous occlusions and independent CT findings suggesting life-threatening events. Korean J Radiol. 14(1): 38-44.

11. Moschetta M, Telegrafo M, Rella L, Stabile Ianora AA, et al. (2014). Multi-detector CT features of acute intestinal ischemia and their prognostic correlations. World $\mathrm{J}$ Radiol. 6(5): 130-38.

12. Van den Heijkant TC, Aerts BA, Teijink JA, Buurman WA, et al. (2013). Challenges in diagnosing mesenteric ischemia. World J Gastroenterol. 19(9): 1338-1341.

13. Jun KW, Kim MH, Park KM, Chun HJ, et al. (2014). Mechanical thrombectomy-assisted thrombolysis for acute symptomatic portal and superior mesenteric venous thrombosis. Ann Surg Treat Res. 86(6): 334-341.

14. Endean ED, Barnes SL, Kwolek CJ, Minion DJ, et al. (2001). Surgical management of thrombotic acute intestinal ischemia. Ann Surg. 233(6): 801-808.

15. Fiengo L, Paciotti C, Patrizi G, Venturini L, et al. (2011). Endovascular repair for acute mesenteric ischemia: case report. BMC Geriatr. 11(Suppl 1): A17.

16. Altintoprak F, Arslan Y, Yalkin O, Uzunoglu Y, et al. (2013). Mean platelet volume as a potential prognostic marker in patients with acute mesenteric ischemia-retrospective study. World J Emerg Surg. 8: 49.

17. Romano N, Prosperi V, Basili G, Lorenzetti L, et al. (2011). Acute thrombosis of the superior mesenteric artery in a 39-year-old woman with protein-S deficiency: a case report. J Med Case Reports. 5: 17.

18. Van De Winkel N, Cheragwandi A, Nieboer $\mathrm{K}$, van Tussenbroek F, et al. (2012). Superior mesenteric arterial branch occlusion causing partial jejunal ischemia: a case report. J Med Case Reports. 6: 48. 